\title{
AbaQuim - Um Jogo Educativo para Auxílio na Aprendizagem de Distribuição Eletrônica Química
}

\author{
Francisco Bruno de S. Rocha ${ }^{1}$, Diego P. Rocha ${ }^{1}$, Nayana Bruna N. Monção ${ }^{1}$, \\ Karoline de M. Farias ${ }^{1}$, Ranulfo P. Bezerra Neto ${ }^{1}$, João Guilherme C. Costa ${ }^{1}$, \\ Bruno V. Alves de Lima ${ }^{2}$, André M. Santana ${ }^{1}$ \\ ${ }^{1}$ Departamento de Computação - Universidade Federal do Piauí (UFPI) \\ Campus Universitário Petrônio Portella - 64049-550 - Teresina - PI - Brasil \\ ${ }^{2}$ Instituto Federal do Maranhão (IFMA) \\ Campus São Raimundo das Mangabeiras, Maranhão, Brasil \\ \{f.bruno.rocha, kdemourafarias, ranulfo0s, jgcc9000\}@gmail.com, \\ \{diegoporto, brunanery, andremacedo\}@ufpi.edu.br, brunovicente.lima@ifma.edu.br
}

\begin{abstract}
This paper describes an educational game, the AbaQuim (Ábaco Químico), for mobile devices with android operational system, aiming to stimulate students' learning about chemistry, specifically the electronic distribution of the the periodic table elements. The lack of connection between theory and practice and the lack of tools and strategies that could help to spark the students' interest, contribute to fail on reaching a more significant learning in the subject. AbaQuim proposes an playful approach, based on GBL (Game-Based Learning) pedagogic methodology, as an aid tool for studies on that subject. As a result of this research it is concluded that even with a short contact with the game the students had a significant improvement in their learning.
\end{abstract}

Resumo. Este artigo descreve um jogo educativo, chamado AbaQuim (Ábaco Químico), para dispositivos móveis de sistema operacional Android com a finalidade de estimular alunos ao aprendizado de Química, especificamente a distribuição eletrônica de elementos da tabela periódica. A falta de relação entre a teoria e a prática, e a escassez de ferramentas e estratégias que ajudem a despertar o interesse do aluno contribui para que ele não consiga um aprendizado mais significativo na disciplina. O AbaQuim propõe uma abordagem lúdica, baseada na metodologia pedagógica GBL (Game-Based Learning), de maneira a desenvolver uma ferramenta de auxilio ao estudo desse conteúdo. Como resultado desta pesquisa, conclui-se que, mesmo com o curto contato com o jogo, os alunos tiveram uma melhora significativa em seu aprendizado.

\section{Introdução}

O estudo de Química, como em outra área de exatas, não é atrativo para a maior parte dos alunos [Pereira and Lima 2014]. Muitos não veem interesse algum devido à alegação da dificuldade em torno do aprendizado dos conteúdos estudados [Pereira and Lima 2014]. Segundo [Zanon and Palharini 1995], muitos alunos e alunas demonstram dificuldades em aprender química, nos diversos níveis do ensino, por não perceberem o significado ou a validade do que estudam. Quando os conteúdos não são contextualizados adequadamente, estes tornam-se distantes, assépticos e difíceis, não despertando o interesse e a motivação dos alunos. 
Na última década houve uma ascensão rápida no uso das chamadas Tecnologias da Informação e Comunicação (TIC), e a aplicação dessas tecnologias no âmbito da educação têm se tornado comum. O uso das TIC ampliou as possibilidades de acesso à informação, tornando-se parte integrante de projetos educacionais. Essas mudanças estão fornecendo novas possibilidades de práticas docentes, visando melhorar a qualidade do processo educativo [Elias 2011]. Na última década, a difusão rápida de dispositivos móveis na sociedade, especialmente smartphones e tablets, ofereceu oportunidades de inovação no processo educacional.

A aprendizagem móvel é um novo paradigma educacional, o qual é mais flexível do que a aprendizagem que utiliza computadores tradicionais. Ela permite que qualquer pessoa possa acessar informações e materiais de aprendizagem de qualquer lugar e a qualquer hora [Demirbilek 2010]. Assim, a aprendizagem mediada pela tecnologia móvel é flexível e adaptável, pois professor e aluno não estão vinculados a um espaço de aprendizagem especial, como um laboratório de informática [Earnshaw et al. 2011]. O aumento do uso social dos jogos de entretenimento em dispositivos móveis, aliado com as implantações de novas técnicas de imersão no ensino-aprendizagem corrobora a premissa de que, o aprendizado baseado em jogos educativos móveis é uma abordagem válida para apoiar abordagens formais de aprendizagem [De Freitas 2011].

Neste artigo é apresentado o jogo educativo Ábaco Químico (AbaQuim), que tem como objetivo ensinar distribuição eletrônica e visa instigar estudantes em todos os níveis do ensino a aprender e desenvolver o raciocínio sobre este assunto. $\mathrm{O}$ uso de tecnologias, como jogos de computadores, para auxílio na educação e formação, deu origem a uma metodologia pedagógica conhecida como Aprendizagem Baseada em Jogos (GBL de Game-Based Learning) que tem como objetivo principal o apoio à aprendizagem, a avaliação e análise de alunos e melhorias do ensino [Tang et al. 2009]. E é com base nessa metodologia que o estudo e desenvolvimento deste trabalho se apoia.

Nesta abordagem, o estudante é desafiado a fazer a distribuição eletrônica dos elementos da tabela periódica e, em uma parte separada, a disposição dos spins nos orbitais. Além disso, foi desenvolvido um sistema de pontuação onde é considerado o tempo de resposta e número de erros, que são mostrados ao usuário como mensagens de alerta.

As principais contribuições deste trabalho são, o desenvolvimento de uma aplicação interativa que apresenta como objetivo despertar o interesse de estudantes de maneira lúdica, rápida e eficiente sobre um determinado conteúdo de química. Além disso, após aplicação dos testes de validação, é percebido uma melhora significativa da média dos alunos que se submeteram à pesquisa. Isso mostra que o jogo cumpre seu papel como ferramenta de apoio à aprendizagem, o que será evidenciado com mais detalhes nas próximas seções.

\section{Trabalhos Relacionados}

Jogos educacionais atualmente são muito abordados na literatura para o auxiliar ensino de alguma matéria, ou assunto específico. Segundo [Monsalve et al. 2013] jogos são importantes ferramentas para apoio ao ensino, pois permitem treinar, ensinar, aprender e identificar elementos que não são comuns no modelo tradicional. Além disso, os jogos computacionais tem grande potencial de melhorar os processos no qual estão inseridos, pois podem evoluir e incorporar melhorias de acordo com as necessidades. Na litera- 
VI Congresso Brasileiro de Informática na Educação (CBIE 2017)

Anais do XXVIII Simpósio Brasileiro de Informática na Educação (SBIE 2017)

tura existem diversos exemplos de abordagens que seguem esse modelo educacional, o trabalho de [Morsi and Mull 2015] apresenta um jogo de aventura educacional em 3D para abordar Sistemas de Números, Álgebra Booleana e Combinacional, além de Design Lógico no âmbito dos currículos de Engenharia Elétrica e Computação.

No trabalho [Winter et al. 2016] é desenvolvido o Chair!, um jogo mobile para ensinar a conformação cadeira do ciclohexano a estudantes de química. Em [Battistella et al. 2016] é apresentado um jogo educacional, o SCRUM-Scape, para ensinar SCRUM, uma metodologia para o planejamento de projetos de software. [Ramos and Pimentel 2015] apresenta um ambiente gamificado, o VirtualQ, para a Prática Experimental de Transformações Químicas. [Silva and Rebouças 2011] e [Perry et al. 2012] apresentam jogos com propostas similares ao jogo desenvolvido neste trabalho com o MyQuímica e o Xenubi, respectivamente.

O MyQuímica [Silva and Rebouças 2011] consiste em um jogo educacional de química para dispositivos móveis, onde os alunos são familiarizados com a tabela periódica e com as nomenclaturas químicas. O jogo propõe aos alunos vários desafios relacionados à composição química, onde o jogador interage com uma tabela periódica, arrastando os elementos de determina composto para um tubo de ensaio virtual. À medida que elementos são colocados no tubo de ensaio, a fórmula do composto formado também é mostrada. Concluído o processo de composição da fórmula, o jogador utiliza a opção "Misturar"presente no menu. Ao acertar o desafio, o jogo apresenta informações sobre o composto para que o usuário aprenda mais sobre ele e contextualize-o com o seu cotidiano (Figura 1(a)).

O Xenubi [Perry et al. 2012] trata-se de um jogo de química para computador que realiza o exercício de conhecimento por parte do aluno em relação à intensidade das propriedades dos elementos químicos e da posição em que eles se encontram na tabela periódica. Assim, dois elementos químicos aparecem posicionados em uma Tabela Periódica. O jogador deve analisar a posição dos elementos e escolher qual propriedade química do seu elemento é superior ao elemento do oponente, no caso, o computador. O jogo também fornece dicas ao jogador, esta funcionalidade pode ser acionada a qualquer momento de forma a auxiliar o aluno a fazer uma escolha consciente (Figura 1(b)).

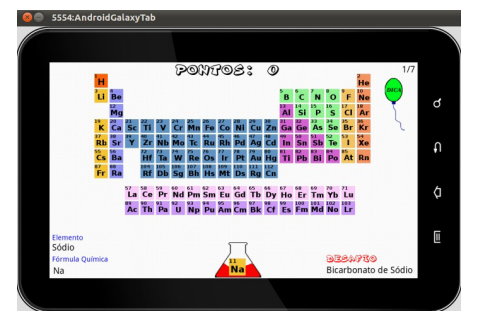

(a) Tela do jogo MyQuímica
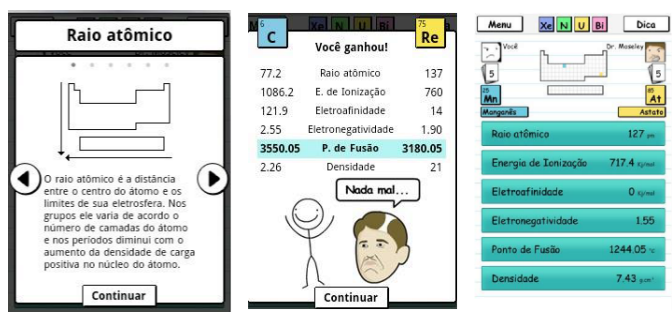

(b) Tela do jogo Xenubi

Figura 1. Exemplos de jogos para o ensino de química.

O AbaQuim auxilia o ensino de uma área específica da química, a distribuição eletrônica de elementos. Assim como o MyQuímica e Xenubi, apresenta a tabela periódica de elementos químicos e as informações gerais de cada elemento. O AbaQuim abrange configuração eletrônica dos elementos, abordando os níveis e subníveis dos elementos através da distribuição eletrônica. Apresenta de forma lúdica mensagens educati- 
VI Congresso Brasileiro de Informática na Educação (CBIE 2017)

Anais do XXVIII Simpósio Brasileiro de Informática na Educação (SBIE 2017)

vas quando o jogador erra em qualquer etapa do processo de distribuição, permitindo consolidação do conhecimento. E também, é dado uma pontuação ao jogador no término da distribuição, incentivando-o sempre jogar novamente para alcançar pontuações maiores.

\section{AbaQuim}

O jogo AbaQuim, desenvolvido neste trabalho, foi projetado para dispositivos móveis com sistema Android, especificamente smartphones e tablets, sendo que para melhor jogabilidade e visualização das informações é recomendado o uso de tablet. O jogo é suportado nas versões Android 2.2, Froyo, até as mais novas versões Android 6.0, Marshmallow. Ele também é adaptável às resoluções maiores que 320x240 que abrange a maioria dos dispositivos móveis existentes hoje no mercado, limitando-se apenas às resoluções abaixo de 480x320, porque não será possível usar os elementos de interação do jogo devido ao seus tamanhos diminutos.

Esta alta taxa de compatibilidade ocorre devido ao uso do motor de jogos 2D código aberto chamado AndEngine escrito em linguagem de programação Java. O motor usa a biblioteca gráfica Open Graphics Library for Embedded Systems (OpenGL ES) que provê uma tecnologia de aceleração gráfica. Para o desenvolvimento deste projeto foi escolhido a plataforma Eclipse com linguagem de programação Java. Mais informações sobre o motor AndEngine em [Gramlich 2010], onde é possível encontrar blog, fórum, documentação e o código fonte.

O método de interação do usuário com o jogo é pela tela de toque do dispositivo. As interações disponíveis por toque são: acionar botões, escolher um elemento da tabela periódica, deslisar subcamada e preencher spins dos orbitais do elemento. A Figura 2 mostra a arquitetura do jogo desenvolvido. Na tela inicial (Figura 3(a)) o jogador pode escolher entre Jogar, Opções e Créditos. Ao selecionar Opções, é possível alterar as configurações do jogo, como o volume, por exemplo. A opção Créditos fornece informações sobre a aplicação e desenvolvedores. E ao selecionar a opção Jogar, é dado início a ciclo do jogo.



Figura 2. Representação da arquitetura do AbaQuim.

Na primeira fase do ciclo, o jogador pode visualizar a Tabela Periódica de elementos (Figura 3(b)) e selecionar, inicialmente, os primeiros elementos de cada família da tabela para visualizar suas informações e realizar a distribuição eletrônica. Todos os outros elementos ficam em estado bloqueado e, a medida que o usuário completa a distribuição de um elemento, o próximo da sua família será desbloqueado. Com o elemento selecionado, é apresentado suas informações principais como demonstrado na Figura 3(c), além disso, sempre que possível, é apresentado uma imagem do elemento em seu estado natural ao lado das informações. 
VI Congresso Brasileiro de Informática na Educação (CBIE 2017)

Anais do XXVIII Simpósio Brasileiro de Informática na Educação (SBIE 2017)

Após a apresentação das informações do elemento, o jogador pode optar por Voltar, e escolher outro elemento da Tabela Periódica, ou Começar. Se for selecionado Começar, o jogador será direcionado para Tela de Distribuição semelhante a (Figura 3(d)). Essa tela foi diretamente inspirada no Ábaco, um objeto inventado na Antiguidade para realização de cálculos matemáticos por meio de deslizamento de pequenas esferas por hastes paralelas. Em vez de cálculos, o usuário fará uma distribuição eletrônica química arrastando os orbitais na ordem correta para a última haste. Devido a essa mecânica, o jogo foi chamado de AbaQuim. O jogador tem que fazer a distribuição eletrônica do elemento selecionado o mais rápido possível evitando erros para conseguir mais pontos no final do jogo.

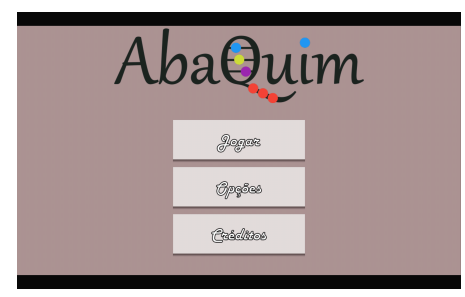

(a) Tela inicial do Jogo.

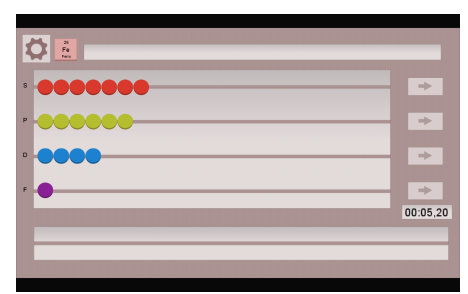

(d) Tela de distribuição (e) eletrônica.
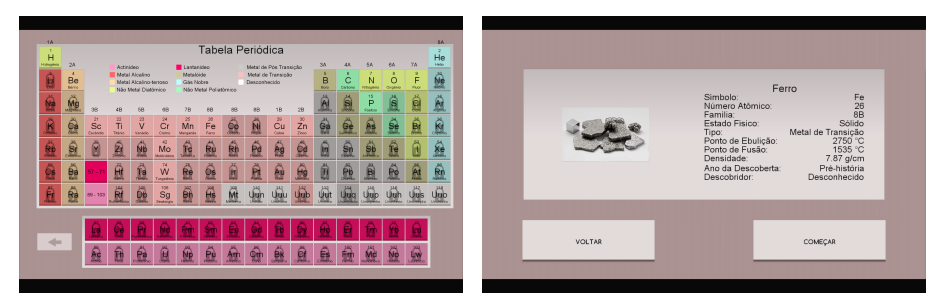

(b) Tabela periódica de elementos.

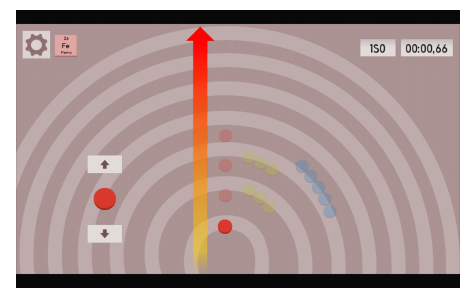

dos spins. (c) Informações do elemento selecionado.

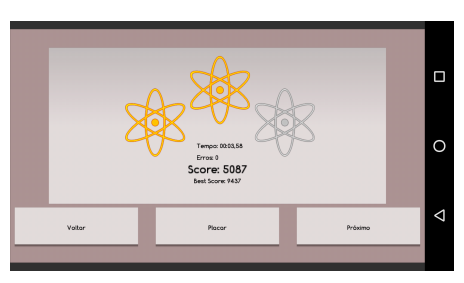

(f) Tela de pontos do jogo.

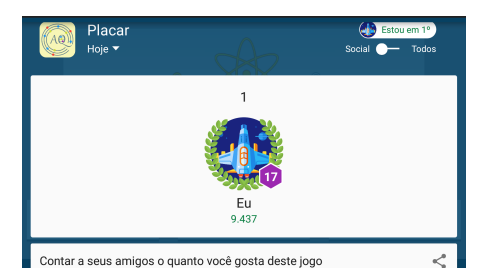

(g) Placar do jogo.

Figura 3. Telas do jogo AbaQuim.

Terminada a Distribuição, o jogador é direcionado para configuração eletrônica química (Figura 3(e) do elemento selecionado. Para jogar, é necessário navegar entre as subcamadas do elemento e colocar os spins na ordem correta. Mensagens de erro aparecerão caso ocorra a disposição incorreta dos spins ou o avanço não-sequencial para outro orbital. Após a correta disposição dos spins, o usuário conclui a iteração e o jogo transita para Tela de Pontuação. Nela é apresentado os pontos totais conseguidos pelo jogador ao decorrer do jogo, também conhecido como score no mundo dos jogos (Figura 3(f)).Nesta mesma tela, é possível acionar o botão placar que redireciona o jogador para um placar geral disponibilizado pelo Google Play(Figura 3(g)). Essa funcionalidade permite classificar os jogadores a partir do seu desempenho em cada elemento jogado, esta estratégia, característica em jogos, permite criar um ambiente de competição entre os jogadores for- 
VI Congresso Brasileiro de Informática na Educação (CBIE 2017)

Anais do XXVIII Simpósio Brasileiro de Informática na Educação (SBIE 2017)

necendo informações estatísticas de seus desempenhos. A seguir, é possível ir para Tabela Periódica e selecionar outro elemento ou ir para o próximo nível reiniciando o ciclo.

\subsection{Testes Funcionais}

Os testes da aplicação foram realizados com sucesso em 3 tablets e 2 smartphones, como descrito na Tabela 1, demonstrando que a aplicação é hábil para rodar em diversos dispositivos.

\begin{tabular}{|c|c|c|c|c|c|}
\hline & $\begin{array}{c}\text { Tablet } \\
\text { Samsung } \\
\text { Galaxy } \\
\text { Tab Pro 10.1 }\end{array}$ & $\begin{array}{c}\text { Tablet } \\
\text { Goldentec } \\
\text { Gt T8270 }\end{array}$ & $\begin{array}{c}\text { Tablet } \\
\text { Multilaser } \\
\text { M7s }\end{array}$ & $\begin{array}{c}\text { Smartphone } \\
\text { Moto } \\
\text { X G2 }\end{array}$ & $\begin{array}{c}\text { Smartphone } \\
\text { Moto } \\
\text { X G2 }\end{array}$ \\
\hline $\begin{array}{c}\text { Tamanho da } \\
\text { Tela }\end{array}$ & $10.1 \mathrm{pol}$ & $7 \mathrm{pol}$ & $7 \mathrm{pol}$ & $4.95 \mathrm{pol}$ & $5.2 \mathrm{pol}$ \\
\hline Resolução & \begin{tabular}{|c|}
$2560 \mathrm{x}$ \\
$1600 \mathrm{px}$
\end{tabular} & $\begin{array}{c}800 \mathrm{x} \\
480 \mathrm{px}\end{array}$ & $\begin{array}{c}1920 \mathrm{x} \\
1080 \mathrm{px}\end{array}$ & $\begin{array}{c}1920 \mathrm{x} \\
1080 \mathrm{px}\end{array}$ & $\begin{array}{c}1280 \mathrm{x} \\
1080 \mathrm{px}\end{array}$ \\
\hline Processador & $\begin{array}{c}\text { Quad-Core } \\
1.9 \mathrm{GHz} \\
\text { Cortex-A15 }\end{array}$ & $\begin{array}{c}\text { Quad-Core } \\
1.5 \mathrm{GHz} \\
\text { Cortex-A7 }\end{array}$ & $\begin{array}{c}\text { Quad-Core } \\
1.2 \mathrm{GHz}\end{array}$ & $\begin{array}{c}\text { Quad-Core } \\
2.3 \mathrm{GHz} \\
\text { Krait } 400\end{array}$ & $\begin{array}{c}\text { Quad-Core } \\
2.5 \mathrm{GHz} \\
\text { Krait } 400\end{array}$ \\
\hline Memória & $2 \mathrm{~GB}$ & $512 \mathrm{MB}$ & $512 \mathrm{MB}$ & $2 \mathrm{~GB}$ & $2 \mathrm{~GB}$ \\
\hline $\begin{array}{c}\text { Placa de } \\
\text { Vídeo }\end{array}$ & $\begin{array}{c}\text { Mali-T628 } \\
\text { MP6 }\end{array}$ & & & Adreno 330 & Adreno 330 \\
\hline $\begin{array}{c}\text { Sistema } \\
\text { Operacional }\end{array}$ & $\begin{array}{c}\text { Android } \\
\text { OS, } \\
\text { v4.4 } \\
\text { (KitKat) }\end{array}$ & $\begin{array}{c}\text { Android } \\
\text { OS, } \\
\text { v4.4 } \\
\text { (KitKat) }\end{array}$ & $\begin{array}{c}\text { Android } \\
\text { OS, } \\
\text { v4.4 } \\
\text { (KitKat) }\end{array}$ & $\begin{array}{c}\text { Android } \\
\text { OS, } \\
\text { v5.0 } \\
\text { (Lolipop) }\end{array}$ & $\begin{array}{c}\text { Android OS, } \\
\text { v6.0 } \\
\text { (Marshmallow) }\end{array}$ \\
\hline
\end{tabular}

\section{Validação e Análise dos Resultados}

A Validação e a Análise dos Resultados objetivam aferir a eficiência do AbaQuim em cumprir o seu objetivo de auxilio ao aprendizado do conteúdo de distribuição eletrônica em química. As seções subsequentes descrevem o teste de validação e sua execução, assim como a análise dos resultados. O processo de validação da proposta foi baseado em [Rodrigues et al. 2015].

\subsection{Validação}

Com a devida autorização dos participantes, por meio da assinatura de um termo de consentimento livre e esclarecido, foram realizadas quatro atividades para validar o aplicativo. As atividades foram devidamente acompanhadas por um profissional bacharel em Química, o qual se responsabilizou pela realização desta validação.

Para a prática do experimento, 43 alunos do $2^{\circ}$ ano do ensino médio do Colégio Técnico de Floriano foram voluntários para a realização dos testes de validação e avaliação do aplicativo.

As etapas que compõem este teste são as seguintes: $1^{\mathrm{a}}$ etapa: Aula de distribuição eletrônica química; $2^{\mathrm{a}}$ etapa: Aplicação de um pré-teste; $3^{\mathrm{a}}$ etapa: Aplicação do Software Educacional AbaQuim e $4^{\text {a }}$ etapa: Aplicação de um pós-teste, seguindo a mesma metodologia do pré-teste. 
VI Congresso Brasileiro de Informática na Educação (CBIE 2017)

Anais do XXVIII Simpósio Brasileiro de Informática na Educação (SBIE 2017)

\subsection{Aula de Distribuição Eletrônica Química}

A primeira etapa consistiu em uma aula com duração de 15 minutos sobre o conteúdo em questão. Tendo como objetivo, relembrar e esclarecer os alunos sobre o tema abordado pela aplicação. Os assuntos ministrados foram especificamente: modelo atômico de Rutherford-Bohr, distribuição eletrônica (ordem energética e geométrica) de átomos e íons, camada de valência e números quânticos.

\subsection{Aplicação do Pré-Teste}

A segunda etapa consistiu em aplicar um pré-teste que serviu de avaliação de conhecimento dos participantes do experimento. O teste abordava os conteúdos ministrados na aula da primeira etapa, onde avaliou-se os conhecimentos de distribuição eletrônica, e disposição de spins. A avaliação apresentou 8 questões sobre distribuição eletrônica e disposição dos spins de alguns elementos da tabela periódica.

Quanto ao teste escrito, os participantes tiveram que responder às questões discursivamente, onde foram registrados os erros e acertos de cada um, a fim de fornecer uma nota de 0 à 10 para comparar com o pós-teste e então avaliar o quanto o aluno evoluiu com uso da ferramenta educacional. Esta etapa teve duração de 20 minutos.

\subsection{Aplicação do Software Educacional AbaQuim}

A terceira etapa consistiu em familiarizar os alunos com o aplicativo por meio do uso de simuladores Android em um laboratório de informática. Cada participante tinha como meta, fazer a distribuição e o preenchimento de spins de pelo menos 10 elementos da tabela periódica em aproximadamente 15 minutos. Vale ressaltar que foi apresentada a instrumentação básica do aplicativo antes da realização do teste.

\subsection{Aplicação Pós-Teste}

A terceira etapa consistiu em aplicar o pós-teste avaliando se o participante adquiriu conhecimento em relação ao pré-teste. Para isso, foi aplicado o mesmo questionário com o objetivo de avaliar se, com o uso da aplicação, o participante, mesmo com o pouco tempo de uso, conseguiu perceber seus erros do pré-teste e conseguiu consertá-los. Além disso, também foi analisado se ele pôde resolver questões que não conseguiu no pré-teste, isto é, avaliar se o participante adquiriu conhecimento no assunto abordado pelo aplicativo.

Depois da etapa pós-teste, foi requerido aos participantes o preenchimento de um formulário online para a avaliação do aplicativo. Neste questionário foram avaliadas as repostas para seguintes perguntas: Qual a nota você daria ao aplicativo?(com avaliação de 0 a 10)(Figura 4(a)); Você se sentiu motivado a aprender o assunto?(sim ou não)(Figura 4(b)) e Você recomendaria este aplicativo como ferramenta de aprendizado?(sim ou não)(Figura 4(c)). Esta etapa durou no total de 25 minutos, onde 20 minutos corresponderam a aplicação do questionário pós-teste e 5 minutos do formulário online.

\subsection{Análise de Resultados}

Levando em consideração as amostras apresentadas nos testes, foram feitas 3 análises que mostram a eficácia do jogo. Na primeira, é destacado a variação nas notas dos alunos no pré-teste. Na segunda, são analisados os alunos com nota menor do que 5 no pré-teste e no pós-teste maior ou igual a 5. E na terceira é analisado a evolução da média dos alunos. 
VI Congresso Brasileiro de Informática na Educação (CBIE 2017)

Anais do XXVIII Simpósio Brasileiro de Informática na Educação (SBIE 2017)

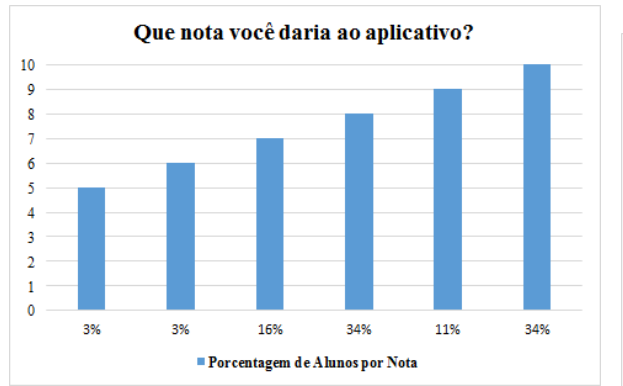

(a)

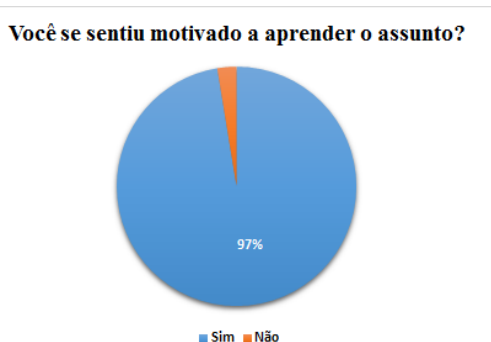

(b)

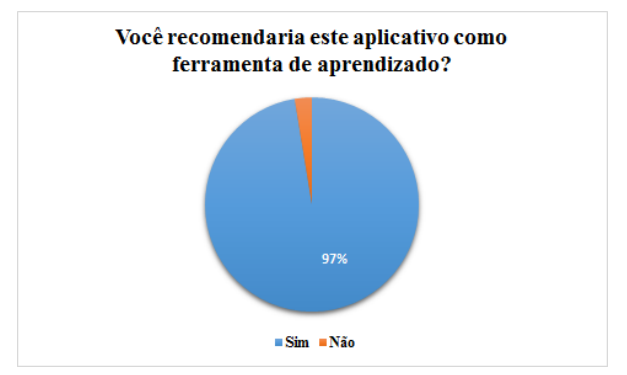

(c)

Figura 4. Avaliação(a), Motivação (b) e Recomendação(c) do Aplicativo.

Na primeira análise, Figura 5, são mostrados os resultados comparativos após aplicação do pré e pós testes, onde são evidenciados os alunos que melhoraram, diminuíram e mantiveram suas notas. A turma teve uma melhora na média das notas que pode ser explicada pela quantidade de alunos que obtiveram uma nota melhor no pós-teste que foi de 34 (79\%) alunos. Nove alunos (21\%) não tiveram alteração na nota e não houve queda de rendimento no pós-teste. A não ocorrência de diminuição de notas no pós-teste mostra que o aplicativo não provocou desentendimento no aluno induzindo-o ao erro, e além disso, ajudou a maioria deles a responderem as questões corretamente.

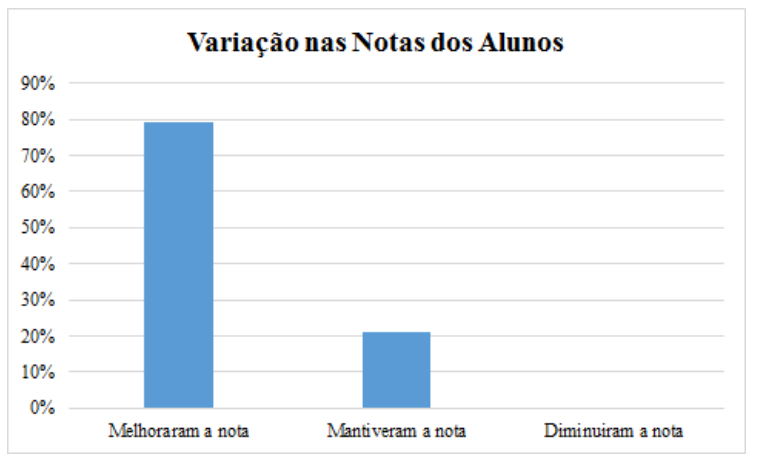

Figura 5. Porcentagem de alunos que melhoram, mantiveram e diminuíram de nota nas aplicações dos testes.

Na segunda análise é avaliado as notas dos alunos considerando a média de aceitação como 5. Dentre os alunos da amostra que não atingiram a nota de aceitação no pré-teste, $11(26 \%)$ alunos passaram da linha de aceitação no pós-teste. Nove (21\%) alunos obtiveram melhora na nota, mas não atingiram a média. Já os alunos que possuíam nota maior ou igual a média de aceitação, 16 (37\%) alunos, mantiveram a nota acima da 
VI Congresso Brasileiro de Informática na Educação (CBIE 2017)

Anais do XXVIII Simpósio Brasileiro de Informática na Educação (SBIE 2017)

média no pós-teste. E sete (16\%) alunos, com notas abaixo da média, mantiveram suas notas nos testes.

Na terceira análise é avaliado a evolução média dos alunos (Figura 6). No préteste os alunos obtiveram uma média de notas de 3,378 com o desvio padrão de 3,374. A turma obteve uma melhora média no pós-teste de $63 \%$ em relação ao pré-teste, com uma média 5,494 e desvio padrão de 3,609. É importante destacar que, 9 (21\%) alunos que obtiveram a nota mínima (0) no pré-teste, após o uso do aplicativo, melhoraram sua nota no pós-teste, mostrando que mesmo alunos com pouco conhecimento sobre o assunto puderam adquiri-lo com o uso da ferramenta. E também, é importante destacar, que alunos que apresentaram um conhecimento moderado sobre o assunto puderam, com o uso da aplicação, atingir a nota máxima (10) da prova, onde a quantidade de alunos que não tiraram 10 no pré-teste e conseguiram fazê-lo no pós-teste foi de 7 (16\%). A partir desses resultados, pode-se concluir que, o jogo tem capacidade de fornecer conhecimento suficiente para que um aluno possa dar início aos estudos sobre distribuição eletrônica, além de aprimorar quem já possui um entendimento do conteúdo.

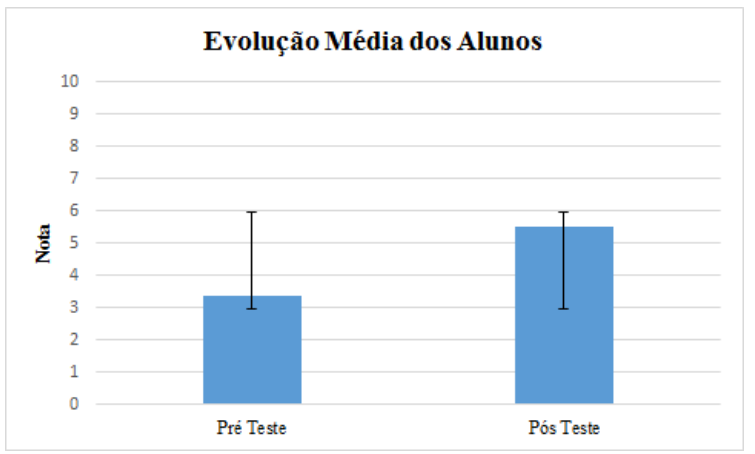

Figura 6. Comparação entre os testes.

\section{Considerações Finais}

A atividade despertou o interesse dos alunos no estudo da configuração eletrônica dos elementos, criando uma dinâmica com a pontuação, de maneira a despertar o interesse do jogador a sempre melhorar seu desempenho a cada elemento jogado. Com a pesquisa de avaliação do aplicativo, observou-se uma boa aceitação quanto a interface e usabilidade, assim, o jogo pode ser considerado como uma ferramenta educacional de fácil uso e jogabilidade. O aplicativo se encontra disponível para download no Google Play ${ }^{1}$.

Pela pesquisa de avaliação dos alunos apresentada nos resultados, mostrou-se que, mesmo em um curto espaço de tempo de uso da aplicação, houve uma melhoria significativa na aprendizagem do aluno sobre o assunto. Isso pode ser evidenciado pelos $79 \%$ dos alunos que melhoraram suas notas após uso do Ábaco Químico, cumprindo a meta do aplicativo e validando-o como uma ferramenta auxiliar educacional de aprendizagem de distribuição eletrônica e disposição dos spins dos elementos da tabela periódica.

Como trabalho futuro, pretende-se implementar um módulo que permita ao professor acompanhar o desempenho do aluno no jogo, incluindo informações sobre as etapas em que ele apresentou dificuldade.

\footnotetext{
${ }^{1}$ https://play.google.com/store/apps/details?id=br.ufpi.dc.raposa. abacodroid
} 
VI Congresso Brasileiro de Informática na Educação (CBIE 2017)

Anais do XXVIII Simpósio Brasileiro de Informática na Educação (SBIE 2017)

\section{Referências}

Battistella, P. E., de Camargo, A. S., and von Wangenheim, C. G. (2016). Scrum-scape: Jogo educacional de role-playing game (rpg) para ensinar scrum. Simpósio Brasileiro de Informática na Educação.

De Freitas, S. (2011). Digital games and learning. Continuum International Publishing Group.

Demirbilek, M. (2010). Investigating attitudes of adult educators towards educational mobile media and games in eight european countries. Journal of Information Technology Education, v.9.

Earnshaw, R. A., Robison, D., and Excell, P. S. (2011). From e-learning to m-learning the use of mixed reality games as a new educational paradigm. International Journal of Interactive Mobile Technologies, v.5.

Elias, T. (2011). Principles for mobile learning. International Review of Research in Open and Distance Learning.

Gramlich, N. (2010). Andengine - um motor para jogos 2d. Disponível em: http: / / www. andengine.org. Acesso em: 27 maio 2015.

Monsalve, E., Werneck, V., and Leite, J. (2013). Incorporando transparência na pedagogia através do uso de jogos para ensino.

Morsi, R. and Mull, S. (2015). Digital lockdown: A 3d adventure game for engineering education. In 2015 IEEE Frontiers in Education Conference (FIE), pages 1-4.

Pereira, D. I. S. and Lima, B. A. T. (2014). Tecnologias de informação e comunicação no ensino de química. Congresso Internacional de Educação e Inclusão, v.1.

Perry, G. T., Kulpa, C. C., and Eichler, M. L. (2012). Lessons from an educational game usability evaluation. International Journal of Interactive Mobile Techonologies.

Ramos, S. and Pimentel, E. P. (2015). Virtualabq ambiente gamificado para a prática experimental de transformações químicas. Simpósio Brasileiro de Informática na Educação.

Rodrigues, M. S., Felix, Z. C., dos Santos Júnior, J. G., and de Almeida, I. R. (2015). Abce bulir: Software para o auxílio no processo de alfabetização e desenvolvimento cognitivo de crianças com síndrome de down. Sociedade Brasileira de Informática na Educação.

Silva, D. L. M. and Rebouças, A. D. D. S. (2011). Myquímica - um jogo para auxiliar no ensino-aprendizagem das nomenclaturas químicas. Simpósio Brasileiro de Informática na Educação.

Tang, S., Hanneghan, M., and El Rhalibi, A. (2009). Introduction to games-based learning.

Winter, J., Wentzel, M., and Ahluwalia, S. (2016). Chairs!: A mobile game for organic chemistry students to learn the ring flip of cyclohexane. Journal of Chemical Education, 93(9):1657-1659.

Zanon, L. and Palharini, E. (1995). A química no ensino fundamental de ciências. Química Nova na Escola - Aprendizado Real. 